\title{
Neural Network-Based Robust Adaptive Beamforming
}

\author{
Xin Song, Jinkuan Wang, Yinghua Han and Dan Tian
}

\begin{abstract}
When adaptive arrays are applied to practical problems, the performances of the existing adaptive algorithms are known to degrade substantially in the presence of even slight mismatches between the actual and presumed array responses to the desired signal. Similar types of performance degradation can occur when the signal array response is known precisely but the training sample size is small. In this paper, we propose a novel neural network approach to robust adaptive beamforming. The proposed algorithm is based on explicit modeling of uncertainties in the desired signal array response and a three-layer radial basis function neural network (RBFNN). In the proposed algorithm, the computation of the optimum weight vector is viewed as a mapping problem, which can be modeled using a RBFNN trained with input/output pairs. Our proposed approach offers fast convergence rate, provides excellent robustness against some types of mismatches and makes the mean output array SINR consistently close to the optimal one. Computer simulation results are presented, which show that the proposed algorithm yields significantly better performance as compared with the existing adaptive beamforming algorithms.
\end{abstract}

\section{INTRODUCTION}

Neural networks have found numerous applications in the field of signal processing [1]-[3], mainly because of their general purpose nature, fast convergence rates, and new VLSI implementations. The aspect of antenna array signal processing focuses on adaptive beamforming. Adaptive beamforming is used for enhancing a desired signal while suppressing noise and interference at the output of an array of sensors. Adaptive beamforming is a ubiquitous task in array signal processing with applications, among others, in radar, sonar, astronomy, and medical imaging and, more recently, in wireless communications [4]-[8]. In particular, the development of robust adaptive beamforming spans over two decades.

When adaptive arrays are applied to practical problems, the performance degradation of adaptive beamforming techniques may become even more pronounced than in the ideal case because some of underlying assumptions on the environment, sources, or sensor array can be violated and this may cause a mismatch between the presumed and actual signal steering vectors. Adaptive array techniques are known to be very sensitive even to slight mismatches of such type that can easily occur in practical situations. In such cases, robust approaches to adaptive beamforming are required. There are several efficient approaches are known to provide an improved robustness against some types of mismatches.

X. Song, J. K. Wang, and Y. H. Han are with School of Information Science and Engineering Northeastern University, Shenyang, 110004, China (phone: +86-335-8066033; fax: +86-335-8051795; email: sxin78916@mail.neuq.edu.cn).

D. Tian is with the School of information, Shenyang University, Shenyang 110044, China (email: dtian@mail.neuq.edu.cn).
The most common is linearly constrained minimum variance (LCMV) beamformer [9], which provides robustness against uncertainty in the signal look direction. To account for the signal steering vector mismatches, additional linear constraints (point and derivative constraints) can be imposed to improve the robustness of adaptive beamforming [10], [11]. But, the beamformers lose degrees of freedom for interference suppression. Recently several other techniques addressing this type of mismatch [12], [13] have been developed. However, they can not provide robustness against other types of mismatches caused by array perturbations, array manifold mismodeling, signal wavefront distortions, and source local scattering, as well as other effects [14][16]. Diagonal loading [17] has been a popular approach to improve the robustness of the adaptive beamforming algorithms. However, a serious drawback of the approach is that there is no reliable way to choose the diagonal loading factor. From the above brief review, it is clear that these approaches can not be expected to provide sufficient robustness improvements.

Neural network, using simple addition, multiplication, division, and threshold operations in the basic processing element, can be readily implemented in analog VLSI. Neural network methods possess such advantages as general purpose nature, nonlinear property, massive parallelism, adaptive learning capability, generalization capability and fast convergence rates. Neural network method is typically used in two steps: training phase and performance phase. Neural network is first trained with known input/output pattern pairs. It can be implemented off-line, although a large training pattern set is required for network training. After the training phase, it can be used directly to replace the complex system dynamics.

Motivated by these inherent advantages of the neural network, this paper presents the development of a neural-network-based robust adaptive beamforming algorithm, which treats the problem of computing the weights of an adaptive array antenna as a mapping problem. Our proposed algorithm provides excellent robustness against some types of mismatches and the small training sample size, enhances the array system performance under nonideal conditions. Computer simulations demonstrate a visible performance gain of the proposed algorithm over other conventional adaptive beamforming algorithms.

\section{PROBLEM FORMULATION}

\section{A. Mathematical model}

Consider a uniform linear array with $M$ omnidirectional sensors spaced by the distance $d$ and $D$ narrowband incoherent plane waves, impinging from directions 
$\left\{\theta_{0}, \theta_{1}, \cdots, \theta_{D-1}\right\}$. The observation vector is given by

$$
\begin{aligned}
\mathbf{X}(k) & =\mathbf{s}(k)+\mathbf{i}(k)+\mathbf{n}(k) \\
& =s_{0}(k) \mathbf{a}+\mathbf{i}(k)+\mathbf{n}(k),
\end{aligned}
$$

where $\mathbf{X}(k)=\left[x_{1}(k), x_{2}(k), \ldots, x_{M}(k)\right]^{T}$ is the complex vector of array observations, $s_{0}(k)$ is the signal waveform, $\mathbf{a}(k)$ is the signal steering vector, and $\mathbf{i}(k)$ and $\mathbf{n}(k)$ are the interference and noise components, respectively. The output of a narrowband beamformer is given by

$$
y(k)=\mathbf{W}^{H} \mathbf{X}(k),
$$

where $\mathbf{W}=\left[w_{1}, w_{2}, \ldots, w_{M}\right]^{T}$ is the complex vector of beamformer weights, and $(\cdot)^{T}$ and $(\cdot)^{H}$ stand for the transpose and Hermitian transpose, respectively. The signal-tointerference-plus-noise ratio (SINR) has the following form:

$$
\mathrm{SINR}=\frac{\mathbf{W}^{H} \mathbf{R}_{\mathbf{s}} \mathbf{W}}{\mathbf{W}^{H} \mathbf{R}_{\mathbf{i}+\mathbf{n}} \mathbf{W}},
$$

where

$$
\begin{gathered}
\mathbf{R}_{\mathbf{s}}=\mathrm{E}\left\{\mathbf{s}(k) \mathbf{s}^{H}(k)\right\}, \\
\mathbf{R}_{\mathbf{i}+\mathbf{n}}=\mathrm{E}\left\{(\mathbf{i}(k)+\mathbf{n}(k))(\mathbf{i}(k)+\mathbf{n}(k))^{H}\right\},
\end{gathered}
$$

are the $M \times M$ signal and interference-plus-noise covariance matrices, respectively, and $\mathrm{E}\{\cdot\}$ denotes the statistical expectation.

Typically, the adaptive beamformer weights are computed in order to optimize the performance in terms of a certain criterion. Although several criteria can be used, we limit our consideration by the output SINR criterion which is rewritten as [18]

$$
\operatorname{SINR}=\frac{\sigma_{s}^{2}\left|\mathbf{W}^{H} \mathbf{a}\right|^{2}}{\mathbf{W}^{H} \mathbf{R}_{\mathbf{i}+\mathbf{n}} \mathbf{W}},
$$

where $\sigma_{s}^{2}$ is the signal power. The problem of finding the maximum of (6) is equivalent to the following optimization problem

$$
\min _{\mathbf{W}} \mathbf{W}^{H} \mathbf{R}_{\mathbf{i}+\mathbf{n}} \mathbf{W} \quad \text { subject to } \quad \mathbf{W}^{H} \mathbf{a}=1 .
$$

From (7), the following solution can be found for the optimal weight vector

$$
\mathbf{W}_{\mathrm{opt}}=\frac{\mathbf{R}_{\mathbf{i}+\mathbf{n}}^{-1} \mathbf{a}}{\mathbf{a}^{H} \mathbf{R}_{\mathbf{i}+\mathbf{n}}^{-1} \mathbf{a}} .
$$

Inserting (8) into (6), we obtain that the optimal SINR is given by

$$
\operatorname{SINR}_{\mathrm{opt}}=\sigma_{s}^{2} \mathbf{a}^{H} \mathbf{R}_{\mathbf{i}+\mathbf{n}}^{-1} \mathbf{a} .
$$

Equation (9) gives an upper bound on the output SINR (6).

\section{B. Sample Matrix Inversion (SMI) Algorithm}

In practical applications, the exact interference-plus-noise covariance matrix $\mathbf{R}_{\mathbf{i}+\mathbf{n}}$ is unavailable. Therefore, the sample covariance matrix

$$
\hat{\mathbf{R}}=\frac{1}{N} \sum_{i=1}^{N} \mathbf{X}(i) \mathbf{X}^{H}(i)
$$

is used instead of $\mathbf{R}_{\mathbf{i}+\mathbf{n}}$, where $N$ is the number of snapshots available. This yields the generalized version of the wellknown sample matrix inversion (SMI) algorithm [19]

$$
\mathbf{W}_{\text {SMI }}=\hat{\mathbf{R}}^{-1} \mathbf{a} \text {. }
$$

When the signal component is present in the data snapshots, the use of the sample covariance matrix $\hat{\mathbf{R}}$ instead of the true interference-plus-noise covariance matrix $\mathbf{R}_{\mathbf{i}+\mathbf{n}}$ affects the performance of the SMI algorithm dramatically. Furthermore, in this case the SMI algorithm does not provide sufficient robustness against a mismatch between the presumed and actual spatial signature vectors.

\section{Loaded sample matrix inversion (LSMI) algorithm}

One of the most popular robust approaches is the loaded SMI (LSMI) algorithm, which attempts to improve the robustness of the SMI technique against an arbitrary spatial signature mismatch by means of diagonal loading of the sample covariance matrix [20]. The essence of the LSMI algorithm is to replace the conventional sample covariance matrix $\hat{\mathbf{R}}$ by the so-called diagonally loaded covariance matrix

$$
\hat{\mathbf{R}}_{\mathrm{dl}}=\zeta \mathbf{I}+\hat{\mathbf{R}},
$$

where $\zeta$ is a diagonal loading factor and $\mathbf{I}$ is the identity matrix. So that, we can write the LSMI weight vector in the following form

$$
\mathbf{W}_{\mathrm{LSMI}}=\hat{\mathbf{R}}_{\mathrm{dl}}^{-1} \mathbf{a}=(\zeta \mathbf{I}+\hat{\mathbf{R}})^{-1} \mathbf{a} .
$$

Although the LSMI algorithm can improve the performance of the SMI algorithm in scenarios with an arbitrary steering vector mismatch, this improvement is not significant. Because the LSMI algorithm exploits the presumed steering vector, its performance degrades when the norm of the error vector is large. Furthermore, the optimal choice of the parameter $\zeta$ depends on unknown scenario parameters and, therefore, the proper choice of $\zeta$ represents a serious problem in practical applications.

\section{Neural Network-Based Robust Adaptive BEAMFORMING}

\section{A. Robust Adaptive Beamforming}

To account for mismatches (including signal steering vector mismatches and more general types of mismatches), we develop a novel approach to robust adaptive beamforming (RAB) that provides an improved robustness against different types of mismatches and the small training sample size.

In practical applications, we assume that the norm of the steering vector distortion $\Delta$ can be bounded by some known constant $\varepsilon>0,\|\boldsymbol{\Delta}\| \leq \varepsilon$. Then, the actual signal steering vector belongs to the set

$$
\boldsymbol{\Phi}(\varepsilon)=\{\mathbf{b} \mid \mathbf{b}=\mathbf{a}+\boldsymbol{\Delta}, \quad\|\boldsymbol{\Delta}\| \leq \varepsilon\} .
$$

The array response should not be smaller than one, i.e.,

$$
\left|\mathbf{W}^{H} \mathbf{b}\right| \geq 1 \text { for all } \mathbf{b} \in \mathbf{\Phi}(\varepsilon) .
$$


According to [21], we can rewrite the optimization problem (7) as

$$
\min _{\mathbf{W}} \mathbf{W}^{H} \mathbf{R W} \quad \text { subject to } \quad \mathbf{W}^{H} \mathbf{a}=\varepsilon\|\mathbf{W}\|+1,
$$

where $\mathbf{R}=\mathrm{E}\left[\mathbf{X}(i) \mathbf{X}^{H}(i)\right]$ denotes the theoretical covariance matrix of the array output vector.

We can also consider the robust adaptive beamforming problem (16) by including other types of mismatches [22]. A meaningful expression of (16) can be written as

$$
\begin{aligned}
& \min _{\mathbf{W}} \max _{\left\|\boldsymbol{\Delta}_{1}\right\| \leq r} \mathbf{W}^{H}\left(\mathbf{R}+\boldsymbol{\Delta}_{1}\right) \mathbf{W} \\
& \text { subject to } \mathbf{W}^{H} \mathbf{a}=\varepsilon\|\mathbf{W}\|+1 .
\end{aligned}
$$

To solve (17), we can first solve the simpler problem

$$
\max _{\boldsymbol{\Delta}_{1}} \mathbf{W}^{H}\left(\mathbf{R}+\boldsymbol{\Delta}_{1}\right) \mathbf{W} \quad \text { subject to }\left\|\boldsymbol{\Delta}_{1}\right\| \leq r .
$$

Rewriting this problem as

$$
\min _{\boldsymbol{\Delta}_{1}}-\mathbf{W}^{H}\left(\mathbf{R}+\boldsymbol{\Delta}_{1}\right) \mathbf{W} \quad \text { subject to }\left\|\boldsymbol{\Delta}_{1}\right\| \leq r .
$$

From the linearity of this objective function, the inequality constraint in (19) can be replaced by the equality constraint

$$
\left\|\boldsymbol{\Delta}_{1}\right\|=r .
$$

The solution to (19) can be obtained using Lagrange multiplier method by minimizing the function

$$
\Psi\left(\boldsymbol{\Delta}_{1}, \lambda\right)=-\mathbf{W}^{H}\left(\mathbf{R}+\boldsymbol{\Delta}_{1}\right) \mathbf{W}+\lambda\left(\left\|\boldsymbol{\Delta}_{1}\right\|^{2}-r^{2}\right) .
$$

Computing the gradient of (21) and equating it to zero yields

$$
\boldsymbol{\Delta}_{1}=\frac{\mathbf{W W}^{H}}{2 \lambda} \text {. }
$$

Inserting (22) into (20) and taking into account that by definition $\lambda \geq 0$, we can obtain

$$
\lambda=\frac{\|\mathbf{W}\|^{2}}{2 r} .
$$

Substituting (23) into (22), we have that the minimum of (21) is achieved at

$$
\boldsymbol{\Delta}_{1}=r \frac{\mathbf{W} \mathbf{W}^{H}}{\|\mathbf{W}\|^{2}} .
$$

Therefore, using (24), we conclude that (18) and, correspondingly, (17) can be rewritten as

$$
\begin{gathered}
\min _{\mathbf{W}} \mathbf{W}^{H}(\mathbf{R}+r \mathbf{I}) \mathbf{W} \\
\text { subject to } \quad \mathbf{W}^{H} \mathbf{a}=\varepsilon\|\mathbf{W}\|+1 .
\end{gathered}
$$

The optimal solution to (25) is obtained by the minimum of the function

$$
\begin{aligned}
L(\mathbf{W}, \lambda)= & \mathbf{W}^{H}(\mathbf{R}+r \mathbf{I}) \mathbf{W}+\lambda\left(\varepsilon^{2} \mathbf{W}^{H} \mathbf{W}-1\right. \\
& \left.-\mathbf{W}^{H} \mathbf{a} \mathbf{a}^{H} \mathbf{W}+\mathbf{W}^{H} \mathbf{a}+\mathbf{a}^{H} \mathbf{W}\right) .
\end{aligned}
$$

Differentiating $L(\mathbf{W}, \lambda)$ with respect to $\mathbf{W}, \lambda$ and setting these partial derivatives equal to zero, we can obtain respectively

$$
\left(\mathbf{R}_{\mathrm{dl}}+\lambda \mathbf{Q}\right) \mathbf{W}=-\lambda \mathbf{a},
$$

and

$$
\mathbf{W}^{H} \mathbf{Q W}+\mathbf{W}^{H} \mathbf{a}+\mathbf{a}^{H} \mathbf{W}-1=0,
$$

where the matrices $\mathbf{R}_{\mathrm{dl}}$ and $\mathbf{Q}$ are defined as

$$
\begin{aligned}
\mathbf{R}_{\mathrm{dl}} & =\mathbf{R}+r \mathbf{I}, \\
\mathbf{Q} & =\varepsilon^{2} \mathbf{I}-\mathbf{a a}^{H} .
\end{aligned}
$$

To solve for the Lagrange multiplier $\lambda$, the equation (27) has an analytical solution given by

$$
\mathbf{W}_{\mathrm{RAB}}=-\lambda\left(\mathbf{R}_{\mathrm{dl}}+\lambda \mathbf{Q}\right)^{-1} \mathbf{a} .
$$

Applying this to (28) yields

$$
\begin{gathered}
f(\lambda)=\lambda^{2} \mathbf{a}^{H}\left(\mathbf{R}_{\mathrm{dl}}+\lambda \mathbf{Q}\right)^{-1} \mathbf{Q}\left(\mathbf{R}_{\mathrm{dl}}+\lambda \mathbf{Q}\right)^{-1} \mathbf{a} \\
-2 \lambda \mathbf{a}^{H}\left(\mathbf{R}_{\mathrm{dl}}+\lambda \mathbf{Q}\right)^{-1} \mathbf{a}-1 .
\end{gathered}
$$

The optimal value of the Lagrange multiplier is then a zero of (32). We can use the eigenvalue / eigenvector decomposition $\mathbf{V} \boldsymbol{\Gamma} \mathbf{V}^{H}=\mathbf{R}_{\mathrm{dl}}^{-1 / 2} \mathbf{Q}\left(\mathbf{R}_{\mathrm{dl}}^{-1 / 2}\right)^{H}$ to obtain the following equation

$$
f(\lambda)=\lambda^{2} \sum_{i=1}^{n} \frac{c_{i}^{2} \gamma_{i}}{\left(1+\lambda \gamma_{i}\right)^{2}}-2 \lambda \sum_{i=1}^{n} \frac{c_{i}^{2}}{\left(1+\lambda \gamma_{i}\right)}-1
$$

where $\mathbf{c}=\mathbf{V}^{H} \mathbf{R}_{\mathrm{dl}}^{-1 / 2} \mathbf{a}$ and $\gamma_{i}$ is known as the generalized eigenvalue of $\mathbf{Q}$ and $\mathbf{R}_{\mathrm{dl}}$.

Since the above equation (31) is not practical for real-time implementation, an adaptive beamforming algorithm must be used to adapt the weights of the array in order to track the desired signal and place nulls in the directions of the interfering signals.

\section{B. The Radial Basis Function Neural Netwok (RBFNN)}

The weight vector of the proposed algorithm is a nonlinear function of the correlation matrix and constraint matrix, and is not suitable for real-time implementation. Therefore, it can be approximated using a suitable architecture such as RBFNN in this paper. The array outputs are preprocessed, and then applied to the RBFNN. The correlation matrix $\mathbf{R}$ is presented to the input layer of the RBFNN, and the vector $\mathbf{W}_{\mathrm{RAB}}$ is produced at the output layer. As it is the case, with most neural network, the RBFNN is designed to perform an input-output mapping, trained with examples $\left(\mathbf{R}^{l} ; \mathbf{W}_{\mathrm{RAB}}^{l}\right) ; l=1,2, \cdots, N_{T}$, where $N_{T}$ stands for the number of examples contained in the training set.

1) The RBFNN Model: The RBFNN is a special threelayered feedforward network, which consists of the input layer, the output layer, and the hidden layer as shown in Fig. 1. In the hidden layer, the nonlinear functions that perform this transformation are usually taken to be Gaussian functions of appropriately chosen means and variances. The weights from the hidden layer to the output layer are identified by following a supervised learning procedure. Assume that the input layer, the hidden layer, and the output layer have $J, L$, $P$ nodes respectively. The network output vector is given by 


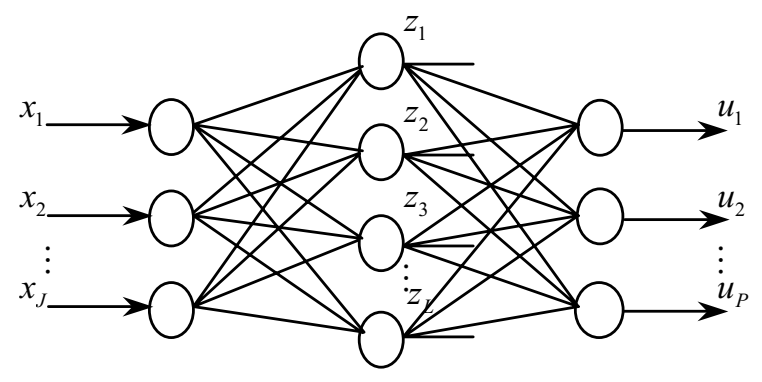

Fig. 1. Structure of a three-layer RBFNN

$$
u_{m}=\sum_{l=1}^{L} \omega_{l, m} e^{\frac{\|\mathbf{x}-\mathbf{c}(l)\|^{2}}{\sigma_{l}^{2}}} \quad(m=1, \ldots, M),
$$

where $\mathbf{x}=\left[x_{1}, x_{2}, \ldots, x_{J}\right]^{T}$ is the input vector to the network, $\mathbf{c}(l)$ and $\sigma_{l}^{2}$ are mean and standard deviation of the $l$ th Gaussian function, $\omega_{l, m}(l=1, \ldots, L ; m=1, \ldots, P)$ is the weight from the $m$ th node in the output layer to the $l$ th node in the hidden layer. Training samples are divided into $L$ classes by $K$-mean learning algorithm, $\mathbf{c}(l)$ is the $l$ th clustering center vector, and $\sigma_{l}^{2}$ is the average distance to the first few nearest neighbors of the means of the other Gaussian functions.

2) Generation of Training Data: By exploiting the symmetry in correlation matrix $\mathbf{R}$, one need only consider either the upper or lower triangular part of the matrix. In our algorithm, the upper triangular half of $\mathbf{R}$ is used to reduce the learning space. The elements in covariance matrix $\mathbf{R}$ are given by

$$
\begin{aligned}
\mathbf{R}_{m l}=\mathrm{E}\left[x_{m} x_{l}^{*}\right]= & \sum_{\substack{i=1\\
}}^{M} \sum_{k=1}^{M} P_{i k} \exp \left\{j 2 \pi f d \left[(l-1) \sin \theta_{k}\right.\right. \\
& \left.\left.-(m-1) \sin \theta_{i}\right]\right\}+\delta_{m l} \sigma_{n}^{2}
\end{aligned}
$$

where $\delta_{m l}$ is the Kronecker delta, $P_{i k}=\mathrm{E}\left[\left(s_{i} s_{k}^{*}\right)\right]$ denotes the source correlation matrix, and $(\cdot)^{*}$ denotes the complex conjugate. An $M \times M$ correlation matrix $\mathbf{R}$ can be rearranged in an $M \times(M-1)$-dimensional vector of real and imaginary parts denoted by $\mathbf{Z}$

$\mathbf{Z}=\left[\mathbf{R}_{12}, \mathbf{R}_{13}, \cdots, \mathbf{R}_{1 M} ; \mathbf{R}_{23}, \cdots, \mathbf{R}_{2 M} ; \cdots ; \mathbf{R}_{(M-1), M}\right]$

then normalize it by its norm, i.e., $\hat{\mathbf{Z}}=\mathbf{Z} /\|\mathbf{Z}\|$.

Produce the required training input/output pairs of the training set, that is, $\left\{\left(\mathbf{R}^{l} ; \mathbf{W}_{\mathrm{RAB}}^{l}\right) ; l=1,2, \cdots, N_{T}\right\}$. In the application, desired sources are located at elevation angels $\theta$ ranging from $-90^{\circ}$ to $90^{\circ}$ to span the field of view of the antenna. Once the RBFNN is trained with a representative set of training input/output pairs, it is ready to function in the performance phase. In the performance phase, the RBFNN produces estimation of the weight vector $\mathbf{W}_{\mathrm{RAB}}$.

3) Performance Phase of the RBFNN: After the training phase is complete, the RBFNN has established an approximation of the desired input-output mapping. In the performance phase, the neural network is expected to generalize, that is, respond to inputs that has never seen before, but drawn from the same distribution as the inputs used in the training set. In the performance phase, the RBFNN produces outputs to previously unseen inputs by interpolating between the inputs used in the training phase.

1) Generate the rearranged correlation matrix $\hat{\mathbf{Z}}$.

2) Present the array output vector at the input layer of the trained RBFNN. The output layer of the trained RBFNN will produce the estimation of the weight vector for the array output.

Unlike the SMI, the least mean-square, or recursive least squares algorithms, where the optimization is carried out whenever the directions of the desired or interfering signals change, in our algorithm, the weight vector of the trained network can be used to produce the optimum weight vector needed to steer the narrow beams of the adaptive array to the directions of desired signals.

\section{Simulation Results}

In this section, we present some simulations to justify the performance of the proposed neural network-based robust adaptive beamforming. We assume a uniform linear array with $M=10$ omnidirectional sensors spaced half a wavelength apart. For each scenario, 100 simulation runs are used to obtain each simulated point. In the training phase, desired sources are located at elevation angels $\theta$ ranging from $-90^{\circ}$ to $90^{\circ}$, with $\Delta \theta=5^{\circ}$. In all examples, two interfering sources are assumed to impinge on the array from the directions of arrival (DOAs) $30^{\circ}$ and $50^{\circ}$, respectively. The diagonal loading factor $\xi=10 \sigma^{2}$ is taken in the LSMI algorithm, where $\sigma^{2}$ is the noise power.

Example 1: Array beampatterns of the algorithms

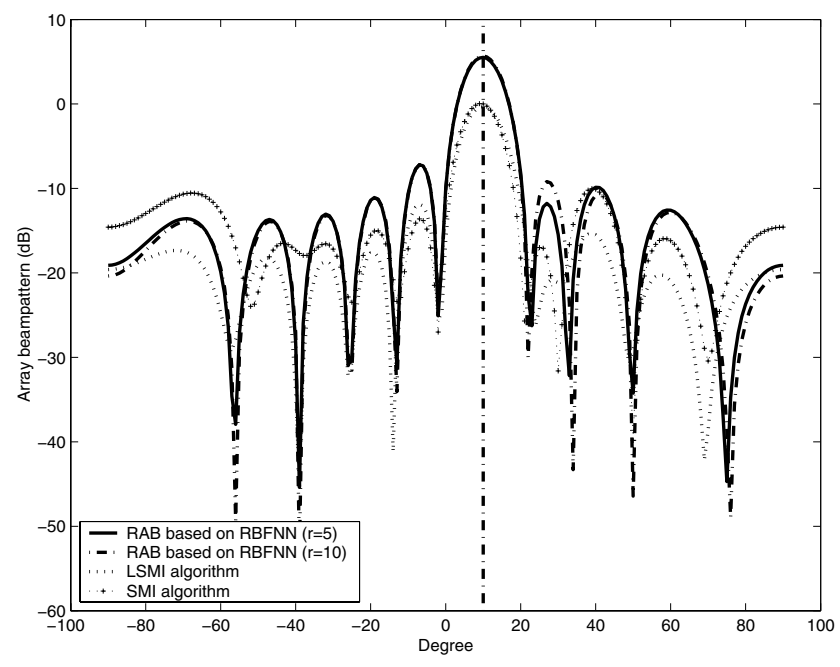

Fig. 2. Comparison of the beampatterns (no mismatch)

In the example, we assume that both the presumed and actual signal spatial signatures are plane waves impinging from the DOAs $10^{\circ}$ and $13^{\circ}$, respectively. Fig. 2 displays the beampatterns of the methods tested for the fixed SNR = 


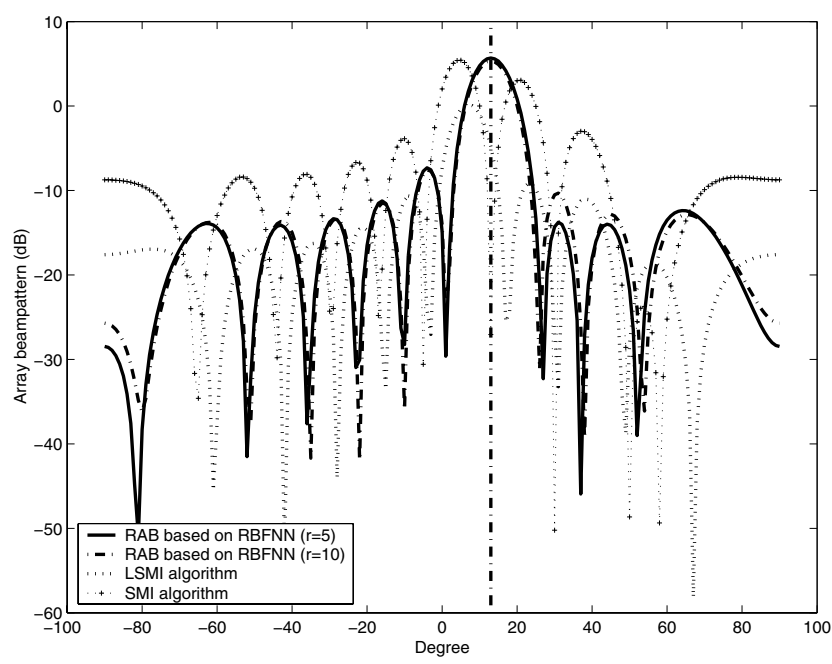

Fig. 3. Comparison of the beampatterns (a $3^{\circ}$ mismatch)

$10 \mathrm{~dB}$ for the no-mismatch case. The vertical line in the figure denotes the direction of arrival of the desired signal. From Fig. 2, we note that the proposed RAB based on RBFNN can adapt the radiation pattern of the antenna to direct narrow beam to the desired signal and nulls interfering sources. Fig. 3 displays the beampatterns of the methods tested for the fixed $\mathrm{SNR}=10 \mathrm{~dB}$ for a $3^{\circ}$ mismatch. The vertical line in the figure denotes the direction of arrival of the actual signal. From Fig. 3, we note that although the beampatterns of the proposed RAB based on RBFNN do not have nulls at the DOAs of the interferences as deep as those of the SMI algorithm, the interferences are sufficiently suppressed by our algorithm.

Example 2: Exactly known signal steering vector

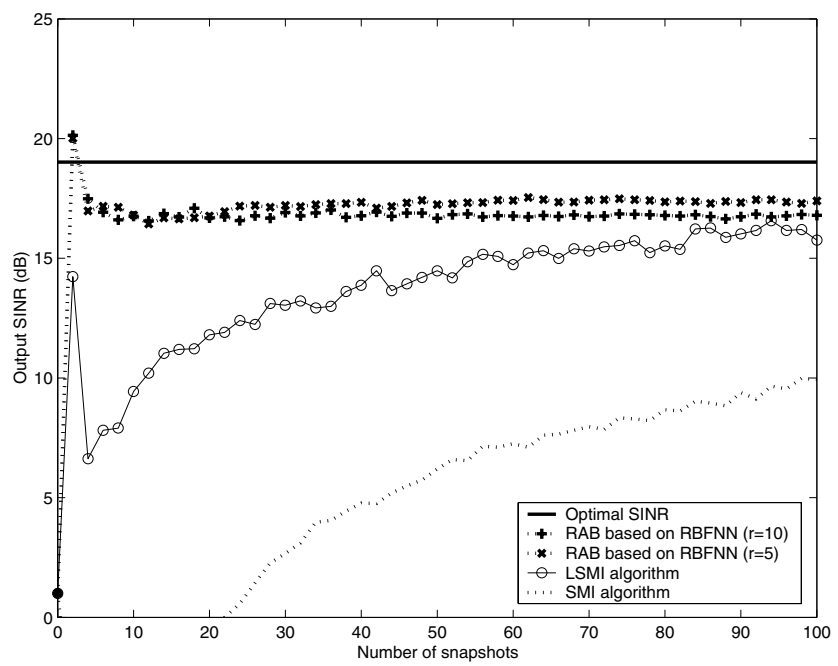

Fig. 4. Output SINR versus $N$

In this example, the plane-wave signal is assumed to impinge on the array from $\theta=10^{\circ}$. Fig. 4 displays the performance of the three methods tested versus the number of snapshots for the fixed SNR $=10 \mathrm{~dB}$. Fig. 5 shows the

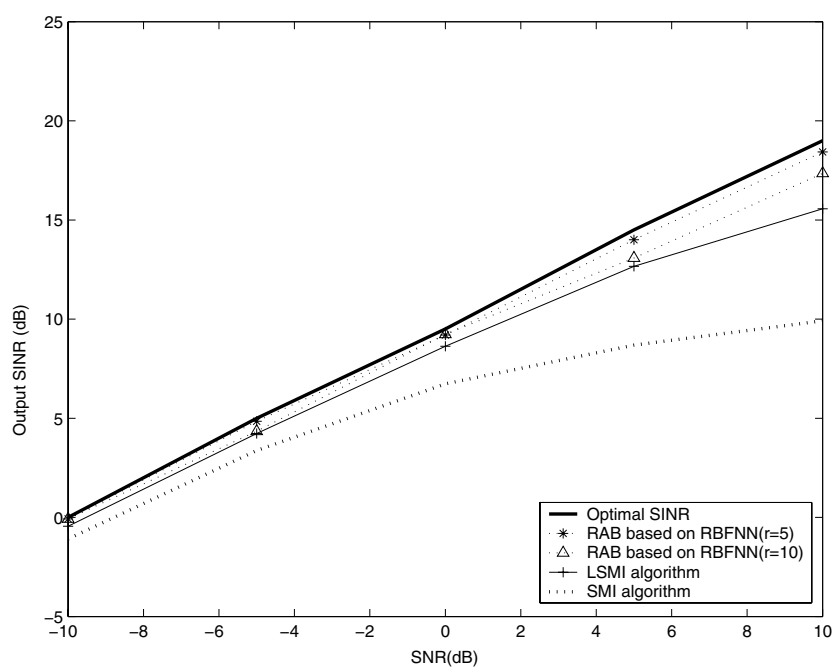

Fig. 5. Output SINR versus SNR

performance of these algorithms versus the SNR for the fixed training data size $N=500$. In the second example, the proposed algorithm offers faster convergence rate than the other algorithms. Note that the performance of the proposed RAB based on RBFNN can outperform that of the other beamforming algorithms.

Example 3: Signal look direction mismatch

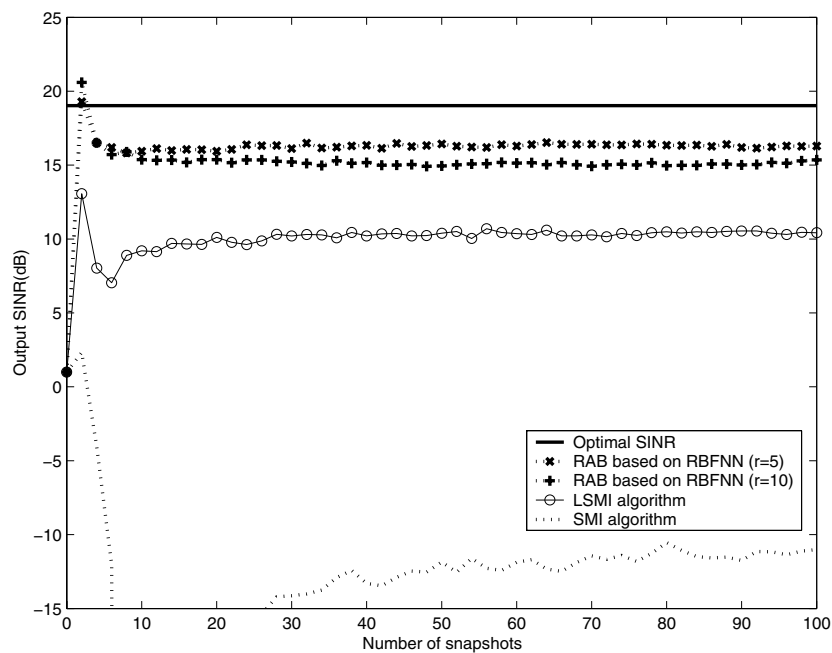

Fig. 6. Output SINR versus $N$

In the example, a scenario with the signal look direction mismatch is considered. We assume that both the presumed and actual signal spatial signatures are plane waves impinging from the DOAs $10^{\circ}$ and $13^{\circ}$, respectively. This corresponds to a $3^{\circ}$ mismatch in the signal look direction. Fig. 6 displays the performance of the three methods tested versus the number of snapshots for SNR $=10 \mathrm{~dB}$. The performance of these algorithms versus the SNR for the fixed training data size $N=500$ is shown in Fig. 7. In this example, SMI algorithm is very sensitive even to slight mismatches that can easily occur in practical situations and 


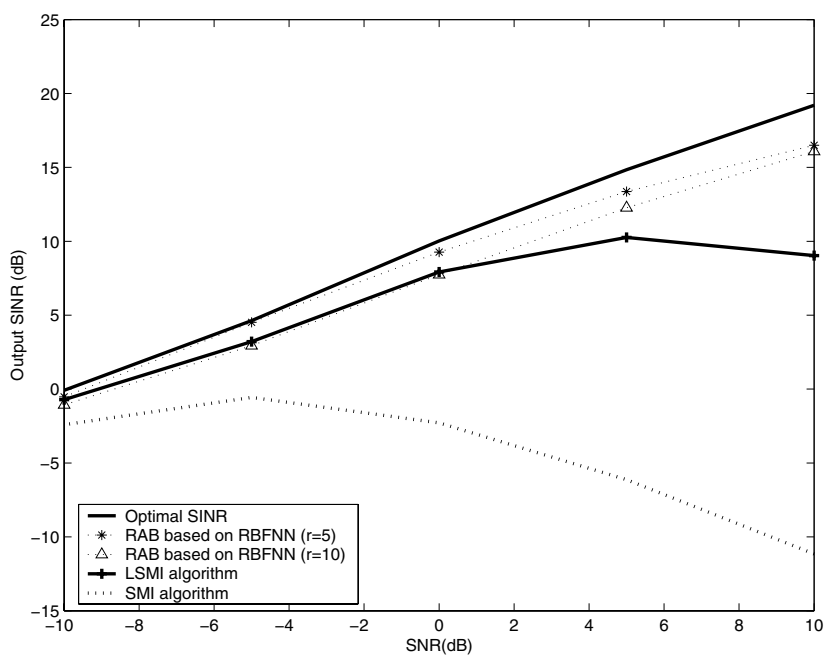

Fig. 7. Output SINR versus SNR

LSMI algorithm can improve the performance of the SMI algorithm. Obviously, the performance of the proposed RAB based on RBFNN is close to the optimal one at all values of the SNR and $N$. The proposed algorithm provides excellent robustness against some types of mismatches and the small training sample size.

\section{Conclusions}

We have shown how to obtain the robust adaptive beamforming based on the RBFNN. The neural network is successful in tracking the desired signal while simultaneously nulling the interference sources. The proposed algorithm is much less sensitive to some types of mismatches and the small training sample size than the other algorithms. Furthermore, the proposed beamformer based on RBFNN consistently enjoys excellent performance because it achieves the values of SINR that are close to the optimal one in a wide range of the SNR and $N$. A number of numerical examples clearly demonstrate that in all examples, the proposed neural network-based robust adaptive beamformer consistently enjoys a significantly improved performance as compared with other beamformers.

\section{ACKNOWLEDGMENT}

This work is supported by Directive Plan of Science Research from the Bureau of Education of Hebei Province, China, under Grant no.Z2004103.

\section{REFERENCES}

[1] A. H. El Zooghby, C. G. Christodoulou, and M. Georgiopoulos, "Performance of radia basis function networks for direction of arrival estimation with antenna arrays," IEEE Trans. Antennas Propagat., vol. 45, pp. 1611-1617, Nov. 1997.

[2] L. Long and L. Y. Da, "Real-time computation of the noise subspace for the MUSIC algorithm," Proc ICASSP 1993, vol. 1, pp. 485-488, Apr. 1993.

[3] P. R. Chang, W. H. Yang and K. K. Chan, "A neural network approach to MVDR beamforming problem," IEEE Trans. Antennas Propagat., vol. 40, pp. 313-322, 1992.
[4] L. E. Brennan, J. D. Mallet, and I. S. Reed, "Adaptive arrays in airborne MTI radar," IEEE Trans. Antennas Propagation, vol. 24, pp. 607-615, Sept. 1976

[5] J. L. Krolik, "The performance of matched-field beamformers with Mediterranean vertical array data," IEEE Trans. Signal Processing, vol. 44, pp. 2605-2611, Oct. 1996.

[6] E. Y. Gorodetskaya, A. I. Malekhanov, A. G. Sazontov, and N. K. Vdovicheva, "Deep-water acoustic coherence at long ranges: Theoretical prediction and effects on large-array signal processing," IEEE $J$. Ocean. Eng., vol. 24, pp. 156-171, Apr. 1999.

[7] L. C. Godara, "Application of antenna arrays to mobile communication, Part II: Beam-forming and direction-of-arrival considerations," Proc. IEEE, vol. 85, pp. 1195-1245, Aug. 1997.

[8] T. S. Rapapport, Ed., Smart Antennas: Adaptive Arrays, Algorithms, and Wireless Position Location, Piscataway, NJ: IEEE, 1998.

[9] R. A. Monzingo and T. W. Miller, Introduction to adaptive arrays. New York: Wiley, 1980.

[10] K. M. Buckley and L. J. Griffiths, "An adaptive generalized sidelobe canceller with derivative constraints," IEEE Trans. Antennas Propagat., vol. AP-34, pp. 311-319, Mar. 1986.

[11] S. Zhang and I. L. Thng, "Robust presteering derivative constraints for broadband antenna arrays," IEEE Trans. Signal Processing, vol. 50, pp. 1-10, Jan. 2002.

[12] L. C. Godara, "Error analysis of the optimal antenna array processors," IEEE Trans. Aerosp. Electron. Syst., vol. AES-22, pp. 395-409, July 1986.

[13] K. L. Bell, Y. Ephraim, and H. L. Van Trees, "A Bayesian approach to robust adaptive beamforming," IEEE Trans. Signal Processing, vol. 48, pp. 386-398, Feb. 2000

[14] J. W. Kim and C. K. Un, "An adaptive array robust to beam pointing error," IEEE Trans. Signal Processing, vol. 40, pp. 1582-1584, June 1992.

[15] N. K. Jablon, "Adaptive beamforming with the generalized sidelobe canceller in the presence of array imperfections," IEEE Trans. Antennas Propagat., vol. AP-34, pp. 996-1012, Aug. 1986.

[16] Y. J. Hong, C. C. Yeh, and D. R. Ucci, "The effect of a finite-distance signal source on a far-field steering Applebaum array-Two dimensional array case," IEEE Trans. Antennas Propagat., vol. 36, pp. 468-475, Apr. 1988.

[17] B. D. Carlson, "Covariance matrix estimation errors and diagonal loading in adaptive arrays," IEEE Trans. Aerosp. Electron. Syst., vol. 24, pp. 397-401, July 1988.

[18] A. B. Gershman, "Robust adaptive beamforming in sensor arrays," Int Journal Electron. Commun., vol. 53, pp. 305-314, Dec. 1999.

[19] I. S. Reed, J. D. Mallett and L. E. Brennan, "Rapid convergence rate in adaptive arrays," IEEE Trans. Aerosp. Electron. Syst., vol. AES-10, pp. 853-863, Nov. 1974.

[20] H. Cox, R. M. Zeskind, and M. H. Owen, "Robust adaptive beamforming," IEEE Trans. Acoust., Speech, Signal Processing, vol. 35, pp. $1365-1376$, Oct. 1987.

[21] S. A. Vorobyov, A. B. Gershman, and Z. Q. Luo, "Robust adaptive beamforming using worst-case performance optimization: a solution to the signal mismatch problem," IEEE Trans. Signal Processing, vol. 51, pp. 313-323, Feb. 2003.

[22] S. Shahbazpanahi, A. B. Gershman, and Z. Q. Luo, "Robust adaptive beamforming for general-rank signal models," IEEE Trans. Signal Processing, vol. 51, pp. 2257-2269, Sept. 2003. 